\section{(b) Sputum.}

$\begin{array}{llllllllllllll}\text { Case No. } & 4 & 5 & 7 & 17 & 18 & 19 & 21 & 22 & 27 & 27 & 29 & 32 & 47\end{array}$

Result... $++++0+++++++0$

$\begin{array}{lllllllllllll}\text { Case No. } & 48 & 49 & 50 & 51 & 52 & 53 & 55 & 56 & 57 & 58 & 59 & 62\end{array}$

Result... $+0+++++++0++$

Total of 25 crses examined, of which 21 cases were positive, making a percentage of $84 \cdot 0$.

(c) Blood Cultures.-Blood cultures were done on some ten of the above cases, and all proved negative as regards the presence of $B$. influenza. In two cases done shortly before death bacterial growths were obtained-in the one a streptococcus and in the other a Gram-negative coccus.

(2) Acute Influenza.-In this instance the posterior naso pharynx only was examined.

$\begin{array}{llllllllllllll}\text { Oase No. } & \ldots & 35 & 36 & 37 & 38 & 39 & 40 & 41 & 42 & 43 & 47 & 45 & 46\end{array}$

Result $\ldots \ldots+00+++++0++0$

Total 12 cases, of which 8 gave positive results (66.8 per cent.)

Altogether 56 unselected cases have been examined to date, and from these the bacillus of influenza was cultured on 42 occasions $(75 \cdot 0$ per cent.).

\section{Disoussion of Results.}

As regards the mere association of the bacillus of influenza with the cases examined the results speak for themselves. The percentage of positive results over the whole series was 74.5 , although in the different groups the figures vary somewhat. In the case of the sputum from bronchopneumonic cases the percentage was 87.5 ; in the postmortem material obtained from a similar series of cases it was 68.3 , while in the naso-pharynx of the acute uncomplicated cases the figure was 66.8. With more prolonged investigations I am sure the number of positive findings of the bacillus of influenza could be increased, as under the conditions of the present time one could ouly spare the minimum amount of time and labour necessary. In addition, there is the probability that a number of the clinical diagnoses may have been at fault as well as the pathological, which would tend to lower the percentage. The later error, however, can be adjusted when histological examination of the post-mortem material has been completed.

Relative Frequenoy of Other Bacteria.

In this articie it will be sufficient to indicate the relative frequency of the other bacteria found in the cases. On the whole, flm examinations have given the impression that Gram-negative bacteria have a tendency to predominate in a good number of the cases, and cultural examinations have confirmed this. Gram-negative diplococci were present on numerous occasions; while cultures showed that some of these belonged to the meningococcus type, more frequently these cocci were of the Pharyngis sicous type. In others large Gram-negative bacilli belonging to the coliform group were present, some of which proved to be the Bacillus proteus and others the Baoullus pneumonice (Friedlander). In addition, especially in the naso-pharynx of acute cases, a small Gram-negative bacillus rather larger than the inflnenza bacillus was isolated. This bacillus was identical with one isolated during the summer epidemic, and was distinctly bipolar in type, with rounded ends. At times the stained bacillus presented an appearance not at all unlike that of the Hacillus pestis.

of the Gram-positive bacteria, the most frequent were staphylococci, both aureus and albus, then streptococci, and lastly pneumococci. In this series a marked feature has been the absence of pneumococci in the various sputa examined, and in not more than two cases were they present in such numbers as to lead one to infer that they played an important part in the process, of four pleuritic fluids examined two gave growths of a fine streptococcus, one a pure culture of pneumococcus, and one a pure growth of $B$. influenze. The slimy condition which the lungs develop in many cases after a short exposure to the air is apparently due to the $B$. protens.

$$
\text { The Role of the Baoillus in Etiology. }
$$

Unlike the influenza bacillus, these various other bacteria do not predominate with anything like the same degree of constancy. These results, however, do not in themselves prove that the $B$. infuenze is the cause of influenza, but only that this bicillus is present in the majority of the cases. One must bear in mind that the mere association of a microorganism with a particular disease does not entitle one to describe it as the cause. For the purpose of illustration it

is sufficient to mention the Baoillus suipestifer and hogcholera, or Bacillus proteus (Strain X.) and typhus fever. The constant presence of the bacillus of influenza is suggestive that it plays an etiological rôle in the production of the condition known as influenza. Conclusive evidence can only be produced as the result of further experimental work.

In acute influenza there is probably an invasion of the respiratory tract by the influenza bacillus, their growth is accompanied by the production of toxic bodies, which injure the mucosa and on absorption produce a general malaise. In the more severe cases the fine bronchi and the alveoli of the lungs are affected. In the latter the walls of the capillaries, as well as the walls of the alveoli, are injured, with the result that a serous exudate takes place. In parts this exudate completely fills up the alveoli, and on this account sections of the affected lung produce a picture strikingly like that produced by phosgene poisoning. The second stage of the disease consists of an infection of the damaged tissues and serous exudate, with one or several of the pathogenic bacteria which are to be found normally in the respiratory tract. Thus a secondary septic bronchopneumonia is set up.

In conclusion, one must admit that the almost constant presence of the bacillus of influenza is suggestive of an etiological rôle, and that prophylactic inoculations against this organism might be successfully employed to combat the disease.

Further, I wish to express my indebtedness to Professor Bulloch for his interest and advice on many points.

\title{
PROVISIONAL NOTES \\ ON THE PATHOLOGY OF THE PRESENT EPIDEMIC.
}

BX P. FILDES, M.B., B.C.,

HONORARY SURGEON-LIEUTENANT CONYANDFR, R.N.V.R.

(Working in the Navy on Behalf of the Medical Research Committee);

S. L. BAKER, M.R C.S., L.R.C.P.,

TEMPORARY GURGEON-IIEUTENANT, R.N.; $A N D$

W. R. THOMPSON, M.SC. CORNELL.

(From the Laboratory, R.N. Hospital, Haslar.)

THE arrival and progress of the influenza pandemic in London appears to have been unexpected among the popula. tion and to have caused a certain extent of dismay. The curious isolation of different communities due to the difficulty of travel and the absence of press facilities has put back the distribution of common knowledge to what it was a hundred years ago. It is possible to read about occurrences in South Africa or Bombay, but news from towns in this country only filters through gradually and often in a distorted form. Thus, while the influenza epidemic has been proceeding sporadically or acutely in Portsmouth for months, the fact has hardly been referred to outside.

Here in Portsmouth the disease has been more or less endemic since the outbreak of "Spanish influenza" in the spring. Pneumonia since that time has been prevalent in the Navy, but since that time it has been certainly more prevalent than usual, and has been the subject of considerable inquiry and study. The number of cases of empyema was remarkable. In these were found not only pneumococci, but also in many cases hæmolytic streptococci, which gave all the ordinary reactions of Streptococous pyogenes.

True general opinion at that time was that the cases were due primarily to either of these organisms or, indeed, to other " respiratory" organisms, since Streptooooons viridans and Streptococcus mucosus were sometimes met with. It was the general impression that "respiratory" bacteria had taken on an increased virulence, owing to the somewhat unsatisfactory weather and the presence of large numbers of young "new entries" in the barracks. The cases were very largely confined to these young "new entries."

The question of influenza as a factor in the disease was discussed and studied, but owing to the complete absence of bacteriological support the causative importance of this bacillus was discredited. 
At the end of August, however, an acute alteration took place in the aspect of the disease. The cases among " new entries" became more and more numerous and the clinical aspect more and more acute. It became obvious that a serious epidemic had broken out. Large numbers of cases of acute pneumonia were admitted to hospital, and among these the death-rate was very high. Localising signs such as empyema became less frequent; indeed, death occurred before these could develop. The outstanding feature of the disease was an acute septicremia of a particularly fulminating type.

At that time the epidemic was very largely confined to the young naval " new entries," and this fact, together with the unusual symptoms, led to all manner of curious suggestions as to its origin. It was even suggested that the disease might be pneumonic plague, while, of course, the foolish seized upon "vaccination" or "inoculation" as the cause, without considering whether either of these protective measures had been already carried out or not.

As the epidemic progressed the age-incidence became widened and all types of men became affected, and finally, as was inevitable, the civil population came under its influence. Clinically, it became more obvious that the acuter symptoms were nearly always preceded by " catarrh," and the question of influenza as the initial infection again came into prominence. Bacteriologically, however, we were unable to obtain any evidence of this ; neither in the sputum nor in throat swabs could we find influenza bacilli. Large numbers of cultures and microscopic examinations, post mortem, were equally unsuccessful. In the trachea, lungs, and blood we now found almost exclusively the same hæmolytic Streptococous pyogeres.

We were therefore inclined to the view that this must be a primary streptococcal pneumonia, although it was obvious that the objections to such a view were serious. On the other hand, we were conscious of the fact that the demonstration of influenza bacilli is at all times difficult, and particularly so under the septic conditions which were before us, and therefore wher Dr. John Matthews ${ }^{1}$ published his technical method for growing $B$. influenze we at once went into the question further.

Among the first cases examined with a digested blood medium comparable to Matthews's, we obtained cultures of $B$. influenzae mixed with large numbers of streptococci from the tonsil after death. In subsequent investigations, using a medium founded upon that of Levinthal (which we refer to as " $K$ " medium) we have found influenza bacilli in so many men suffering from influenza without pneumonia, and in the organs of men dying from pneumonia, that we are of opinion that it is unnecessary to look further than this organism for the cause of the epidemic, the streptococci and the other organisms which have been mentioned being only secondary infections upon lungs already affected by $B$. influenzce.

The difference in the bacterial flora obtained by the use of these media is so remarkable that we are prepared to admit that our earlier conclusions were incorrect, and that $B$. influenze was probably the real cause in most of the cases of pneumonia which have been occurring during the last six months. Under these circumstances it may be an assistance to those who still implicate various streptococci or who are discussing the possibility of an "invisible virus" to have our tentative results so far as they can be carried when half our staff is sick.

\section{Ante-mortem Findings.}

We have examined the naso-pharynx in 15 cases of influenza in whom there were no obvious signs of pneumonia and in 12 have isolated $B$ influenza. Several of these patients later developed a fatal pneumonia, but, unfortunately, no autopsy was permitted. In each case we have made only one culture, and the number of positive results would, undoubtedly, have been much higher if we had made several examinations or if we had been working the whole time with a satisfactory medium. Sometimes the growth has been profuse, but more often a few colonies only develop among the numerous Gram-negative cocci, pneumococci, and streptococci.

We have also examined the naso-pharynx of 71 men who were apparently healthy and obtained 15 positive cultures This finding has an epidemiological interest.

Single samples of sputum have been examined from 106 cases and $B$. influenze bas been found in 45 .
Influenza bacilli can often be cultured from sputa in which it is difficult or impossible to recognise them microscopically. In a small number, however, bacilli were so numerous and pure that they could hardly be missed even by Gram's stain, and we are satisfied that we did not overlook cases of this type in the earlier months of the epidemic. In the great majority of the sputa, including those in which copious growths are obtained, the microscopic picture is inconclusive so far as $B$. influenze is concerned, but pneumococci and streptococci abound as usual. It is true that with a very intense stain, such as that which we call " multicolour blue," a few minute bacilli may be found, often in clumps, but we very seldom get a picture which convinces us that influenza bacilli are present, and the subsequent copious growths are often surprising.

We have made blood cultures from a number of men with pure influenza with negative results, and similar negative results have been obtained with the urine.

$$
\text { Post-mortem Fïndings. }
$$

(a) Maoroscopic pathology.-The post-mortem appearances of a rapidly fatal case will best indicate the type of disease with which we have been dealing here.

Externally: The body of a well-developed muscular young man, aged 18; marked cyanosis of lips and finger-nails; a quantity of blood exuding from mouth. Thorax: 200 c.cm. of deeply blood-stained watery fluid in left pleural cavity. A trace of similar fluid in right chest. The parietal pleura on both sides is injected and shows scattered purpuric fluid. The heart shows nothing remarkable beyond hæmolytic staining of the endocardium; the heart-muscle is firm and appears in good condition. Left lung: The lower lobe is a dark purple colour and shows a fine fibrinous roughening in places; it retains its shape on removal and is heavy; it feels soft and jelly-like, with several firmer areas 2 or $3 \mathrm{~cm}$. in diameter scattered about in the lung substance. The upper lobe is similarly affected in its posterior part, but in the anterior portion it is spongy and feels aerated. On section the lower lobe is dark-red colour and airless, being full of a thin hæmorrhagic exudate which flows out from the cut surface. In the places corresponding to the firmer portions described above there are found solid, dark-red, rather dry areas. Some of these extend to the surface of the lung and resemble infarcts in appearance; others, however, are more or less spherical masses situated centrally in the lung. The lower part of the upper lobe is similarly infiltrated by a thin hæmorrhagic exudate, but the upper part is aerated and shows a congested spongy lung substance. The right lung is less affected than the left, the upper lobe being aerated and showing a red spongy surface. The bronchial tree throughout the lungs is intensely injected and contains bloody fluid which pours out into the trachea. The naso-pharynx, pharynx, larynx, and trachea are much injected. This injection is most marked in the trachea, especially in the lower part, which is a dark purple colour. The tonsils are prominent and injected and contain $a$ few beads of pus. The cervical and paratracheal glands are injected, the latter being a dark purple; the gland below the tracheal bifurca. tion is enlarged and very soft and hæmorrhagic, as are the glands at the hila of the lungs. Abdomen: Peritoneum and intestines appear normal. Spleen not enlarged. On section, slightly swollen red pulp. Kidneys : Cortex rather opaque in appearance, slightly swollen, no hæmorrhages or other abnormalities detected. Suprarenals show dark plumcoloured medullæ, the left being the most affected. Bladder dec.: Nothing remarkable. Brain: Some injection of smaller vessels upon the cerebral cortex. No sigus of meningitis. No hæmorrhage.

The above is a rapidly fatal case showing no reaction to the infection. The patient appears to have been drowned by the exudate into the lungs rather than killed by the septicæmia.

In cases of longer standing there has been, in addition to the hæmorrhagic condition, a marked broncho-pneumonia with pus in the smaller tubes, and sometimes areas of grey hepatisation, as seen in lobar pneumonia. In several cases there has been a purulent bronchitis and peri-bronchitis, the lung presenting a marbled appearance owing to the puralent infiltration of the bronchial walls; the purulent infiltration can also be seen on the surface of the lung as a white peri-lobular network. Empyema is very common, there being, as a rule, a thin yellowish opalescent fluid with a sediment of thicker pus in the most dependent part. Pyopericardium is fr*quent. The spleen shows evidence of reaction, being enlarged and having a swollen, often diffluent, creamy or dark-red pulp. The kidneys often show severe parenchymatous degeneration, but in only one case 
has there been an obvious nephritis. In a few cases dying ten days or so after the onset of the disease the heart muscle appeared considerably affected, being very flabby and having the appearance of parenchymatous degeneration. (b) Bacteriology. - The organism which is immediately responsible for the above pathological picture is a hæmolytic streptococcus; it does not ferment inulin, and grows in long chains as a sediment in broth. It is found in enormous numbers in the lungs and in the pleural and pericardial pus when this is present. From the blood it can be obtained post mortem, usually in pure culture. The coccus, though forming long chains in fluid exudates, often shows diplococcal forms in the tissues, so that it cannot always be recognised as a streptococcus in films. At the beginning of the epidemic this streptococcus was the only organism of importance which could be recovered from the tissues or blood of the cases examined; when, however, the new media became available for the investigation of the cases it was possible to prove, what had already been suspected, that the influenza bacillus was the real cause of the disease.

Previous to the adoption of these media much time was spent examining films from the lung exudates, scrapings from the trachea, \&c., without obtaining any evidence of the presence of the influenza bacillus In the first case in which the bacillus was demonstrated it was found in pus from the enlarged tonsils, and in this it could be seen. with appropriate staining as described elsewhere, in large numbers, chiefly intracellular. Although in this case films from all parts of the respiratory tract were examined, the bacilli could not be detected anywhere else and streptococci in enormous numbers dominated the picture. At the present time we are obtaining cultures of $B$. influenzas from every post-mortem examination upon these pneumonia cases. In the lungs the bacilli are obtained with certainty only from the most healthy parts; the obviously diseased portions are so full of cocci that the bacillus has apparently been exterminated and its detection has generally been impossible. It is obtained fairly constantly from the trachea and bronchi ; in the case of the bronchi those in the healthier parts of the lungs yield the best results. Pus from the deeper parts of the tonsils gives on culture scattered colonies of $B$. influen: $\mathscr{A}$ amongst innumerable streptococci. In one case a culture from blood taken from the jugular vein yielded a few colonies of $B$. influenze and a copious growth of streptococci.

When now these bacteriological findings, ante- and postmortem, are reviewed as a whole, taking into consideration the essentially superficial nature of many of the examinations and the difficulties met with in the preparation of a very delicate medium, it appears justifiable to us to suggest that $B$. influen:ce is very frequently present in the respiratory passages before death, and is present in the lungs and sometimes the blood after death so frequently as to suggest that the disease is constantly associated with and due to a $B$. influenze infection.

The Cultivation of $\mathrm{B}$. influenzæ.

The classical blood agar medium for the growth of influenza bacilli has been practically useless to us under the conditions met with.

We have already mentioned that we first used a digested blood medium akin to Matthews's, but we gave it up in favour of Levinthal's, which is simpler to prepare. We have had no opportunity to study the original description of this medium, but have worked entirely upon the reference to it in the Medical Supplement to the Daily Revirn of the Foreign Press (Oct. 1st, 1918) compiled by the Medical Research Committee. The principle of this medium is the addition of fresh blood to a flask of melted agar in a proportion of 1 in 20. The medium is brought to the boil and the coagulum aseptically filtered off " through gauze or paper."

There are factors in the preparation of this medium which are still obscure. We have several times made up batches which are entirely useless; indeed, we have made up two batches from the same materials and at the same time, and one has been excellent and the other useless. We are almost satisfied that the factor in these cases is the filtration through "gauze or paper." Neither of these substances should ever be used, but glass-wool only, presumably because the paper absorbs the essential "vitamins " (Jordan Lloyd).

We have not yet decided whether the blood must be fresh. We use citrated ox or sheep blood and find this to be satisfactory if collected once a week. Whether a "stock", citrated blood, laked with distilled water and sterilised with chloroform, according to the technique of one of us, is suitable we are not yet certain.

The technique of preparation of " $\mathrm{K}$ " medium is simple.

A flask of ordinary 2 per cent. meat agar is melted and reduced to $55^{\circ} \mathrm{C}$. The agar need not have been "egged " or cleared in any way. One part in 20 of whole blood is then added and the flask placed in a water bath at about $60^{\circ} \mathrm{C}$. The flask is constantly shaken while the water rises to the boil, and when this occurs it is left at rest for 10 minutes at the boiling point. In the meantime a flask carrying an ordinary filter funnel lightly packed half full with glass wool has been autoclaved. The boiled agar-blood mixture is poured through this wool without shaking and, if necessary, left in the steamer. Usually the filtration takes only a minute or two and the medium is then poured aseptically into a number of sterile tubes and sloped. No contaminations need arise during these manipulations.

The effect of this medium upon the growth of $B$. influen:o is very remarkable. This bacillus is familiar to us as producing characteristic pin-point colonies on blood agar. The colonies grow no larger and seldom coalesce. On ordinary agar, the bacillus does not grow at all, but merely by adding the soluble products of a small quantity of boiled blood the growth on agar becomes as profuse and as confluent as the growth of typhoid. The size of the colony depends entirely upon the spacing. The common size is $2 \mathrm{~mm}$., but when colonies are well isolated they often attain no less than $5 \mathrm{~mm}$. The growth recalls that of meningococci on serum agar, but is more transparent. Against a dark background the colonies are also somewhat bluer. They are very easy to distinguish from streptococci or pneumococci. The growth is very readily emulsified to form a stable suspension.

\section{The Identification of $\mathrm{B}$. influenzæ.}

The microscopic appearance of $B$. influenze upon " $\mathrm{K}$ " medium is very characteristic. It is very small and thin. The bacillus is usually so short that it resembles a coccus The longer bacillary forms which are familiar upon blood agar do not develop on " $K$ " medium. Films made from the larger colonies a few days old show a curious degeneration of the bacteria. They have largely broken up into an amorphous débris in which individual bacteria are hardly recognisable, but subcultures from such colonies yield normally profuse growths. The viability of these bacteria appears to be quite prolonged. Even on slope cultures on "K" they live for several days, sometimes for as much as a week, and in capped " stab" cultures in " $K$ " all our strains survive 15 days at least.

The affinity of $B$. infuenze for stains is feeble, and this constitutes one of the chief difficulties in its demonstration. It is, of course, negative to Gram, but the affinity for the countr $r$ stain is so feeble that this method is useless for its detection in pathological material. The stain which we have found most satisfactory is a methylene blue preparation akin to polychrome-methylene blue, which we call "multicolour blue." We made this stain by dissolving $10 \mathrm{~g}$. of methylene blue in a litre of 1 per cent. sodium bicarbonate solution and steaming for two hours. A few drops of this stain are placed upon the slide and this is then heated until steam arises. In the case of a pure culture the stain is then rinsed off and the slide dried, but when dealing with a pathological exudate the slide, after rinsing, should be momentarily placed in alcohol which contains one drop of glacial acetic acid per $100 \mathrm{c.cm}$. It is then rinsed and examined.

There are at present no specific tests which can be applied to identify $B$. influenza. We rely upon the morphological appearance and the growth on blood agar, with an absence of growth on ordinary agar. Most strains grow with the typical discrete, pin-point colonies on blood agar, but some develop rather larger confluent colonies up to $1 \mathrm{~mm}$. The bacillus is, of course, non-motile. Fermentation reactions are now being studied and attempts are being made to prepare agglutinating serum. Rabbits will tolerate very large doses, even two slope cultures of living bacilli.

\section{Prophylaxis of Infuenza.}

It appears to us that the only practical measure which is likely to be effective to control the present epidemic is a prophylactic vaccine treatment. In instituting such a measure it is necessary to decide whether it is more profitable to attempt to prevent the onset of influenza or to 
prevent the complications which are the cause of death. On all gronnds we consider that the former is the more satisfactory aim, since if the influenza can be prevented the complications will not arise. Therefore, we consider that $B$. influenza should be the main basis of the vaccine. We have already prepared large quantities of this vaccine and have experimented with it on volunteers.

The question of the dose to be employed must be governed by several factors. It must not be so large that a danger of a pronounced negative phase will arise, because this would be dangerous when the epidemic is already present and sources of infection are universal. Neither must it be too small to be effective. Further, it is undesirable to produce marked local reactions, since these would create too much dislocation in the carrying on of employment. We are not in favour of giving two doses only, because if these are large enough to be effective a negative phase may follow. We therefore subdivide the first dose into two and give three.

The spacing of doses which we have adopted for the moment is fairly short. The first dose is given on the first day and the others on the fourth and eighth. The quantities given are 50 million, 166 million, and 500 million. The second dose gives rise to some local reaction, and it is possible that the third will have to be reduced.

It has been decided that these vaccines shall be used only upon volunteers, and, since the allegations which have already appeared in certain sections of the press will probably militate against men coming forward, we wish the immediate effects of the vaccine to be as mild as possible otherwise we are afraid that this process, which may well be followed by excellent results, will be discredited and never receive an adequate trial.

\section{THREE CASES OF MALIGNANT DISEASE OF THE UPPER EXTREMITY.}

BY J. W. GEARY GRANT, F.R.C.S. ENG., ACTING ASSISTANT SURGEON, KING EDWARD VII, HOSPITAL, CARDIFF.

THE following cases of malignant disease in the region of the shoulder present, I think, some points of interest.

\section{Case of Berger's Operation.}

The first case, one in which several limited operations had been performed for an epithelioma of the hand, where ultimately the disease had extensively involved the axillary glands and lymphatic vessels, demonstrates the advantages which Berger's operation gives in sweeping away, without a difficult, tedious, and possibly incomplete dissection of the axilla, all possibly infected tissues.

CASE 1.-Patient aged 43. In 1894 suffered from abscesses about both ankles, presumably tubercular. Various operations were performed at different hospitals, and in 1898 a sore formed on the back of the left thumb, which he attributes to a scratch becoming infected whilst dressing his foot. In 1902 the left foot was amputated at Bristol by Mr. R. G. P. Lansdown, and he has had no further trouble with the lower extremities. The sore on the thumb refused to heal, and in October, 1907, X ray treatment was begun and continued at the various places where he resided for over a year. In all, some 50 exposures were given. In February, 1909, he consulted me, and as far as I remember now, there was an indolent ulcer with smooth red surface on the terminal phalanx of the left thumb on its dorsal aspect, the surrounding skin showing numerous minute telangiectases. The sore healed under applications of scarlet red ointment. In October, 1913, it again broke down, and in May, 1914, as it showed no signs of healing, I amputated at the interphalangeal juint by a palmar flap. He had no further trouble until October, 1916, when a blister formed at the end of the stump. Early in 1917 it showed the obvious appearance of the epitheliomatous ulcer, and was amputated through the carpo-metacarpal joint. Recurrence promptly followed in the scar, and a hard cord was found running up the lower part of the palmar aspect of the forearm on the radial side, which on examination was found to be a lymphatic vessel filled with carcinoma cells. The original ulcer on examination was a squamous-celled carcinoma. I then amputated the forearm just below the elbow, removing all the fat and the epitrochlear gland. Glands from the axilla and the cepbalic gland, removed for examination, showed no signs of malignancy. In May, however, glands were felt in the axilla. He was admitted to the King
Edward VII. Hospital and operated on with the intention of clearing the axilla, but as soon as an incision was made it was obvious that this was impossible. There was an extensive glandular involvement and a large thrombosed lymphatic vessel the size of a large crow-quill was found running by and adberent to the axillary vein. His consent to amputation had not been obtained, and the wound was closed. I felt that a Berger's operation held out the best chance of a radical operation and I decided to perform a fore quarter amputation.

Three days later, under open ether, and after a preliminary injection of morphia, I made an incision over the clavicle and resected the middle third with a Gigli's saw. The pectoralis major was separated and the subclavius divided and the neuro-vascular bundle exposed. The artery was ligated at the junction of the axillary with the subclavian (this was lower than it should have been). The arm was elevated to empty it of blook, and the vein secured at the same level. The three cords of the brachial plexus were

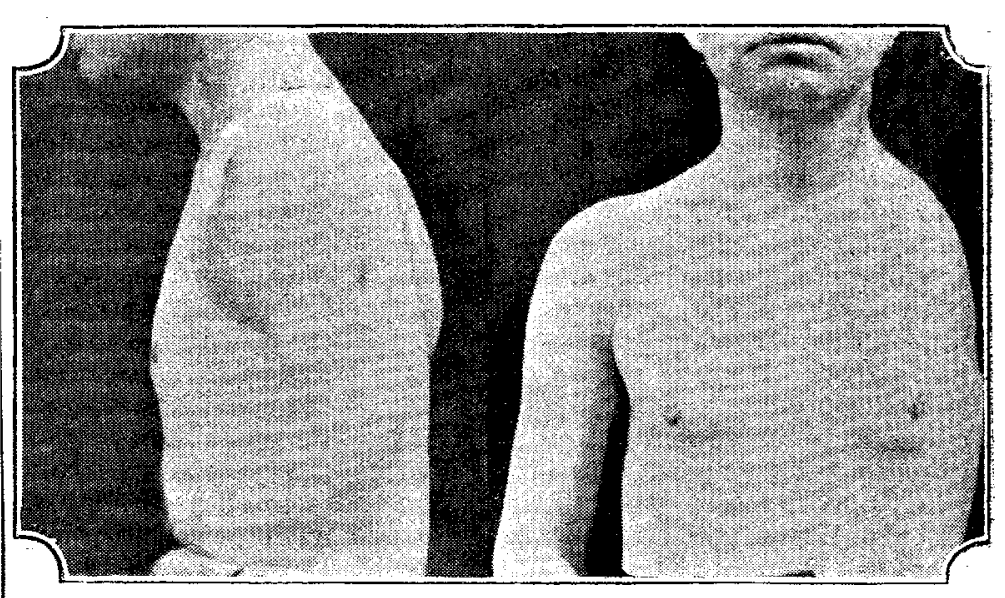

FrG. 1.-Shows smooth rounde outline of shoulder with almost linear scar, well on trout of chest owing to absence of pectoro-axillary flap.

injected with a 4 per cent. solution of novocaine, and after a few minutes divided. The original incision was carried out along the acromion and spine, and then down to the posterior axillary fold. This was joined by another passing from the mid-point of the clavicle in a curve with the concavity ontwards so as to leave practically no anterior flap, the object being to get as far away as possible from the infected axillary contents. Similarly, the pectorals were divided well inwards and reflected with the fascia over the serratus magnus, which was divided a little further out. The cervico-dorsal flap was then reflected, the trapezius, levator anguli, and muscles attached to the posterior border of the scapula divided, the transversalis cervicis and suprascapular arteries secured and the limb removed. In spite of the absence of the pectoro-axillary flap there was ample skin covering. There was very considerable shock, which was treated by continuous rectal saline and the usual methods, and the next day his condition was very good. The wound healed by first intention.

A month later, glands having been found in the neck, an incision was made from the mastoid to the clavicle and outwards to the trapezius, which was partially reflected, and the whole of the fat and glands removed en bloc. These showed evidence of infiltration with carcinoma. At the end of August a small lump was found near the anterior border of the trapezius and was removed. From June to the present date he received 25 exposures to $X$ rays. So far no further signs of the disease have manifested themselves, and he has been for the past six months carrying on his ordinary duties. Fig. 1 shows his appearance.

\section{Case of Amputation at the Shoulder.}

About the same time I had the following case in hospital.

CASE 2.-Patient had a very extensive carcinomatous ulcer arising in the scar of a large burn involving the greater part of the skin of the right upper arm. I amputated at the shoulder, having to make irregular flaps extending on to the chest, as the scar trenched on the axilla and chest wall. The skin covering was not adequate, and part of the wound healed by granulation. The difficulty of feeling sure the axilla was completely cleared in this case afforded a marked contrast with the last, where everything was swept away en bloc, without a tedious dissection. Here one had to think about getting flaps to cover the stump of the scapula and acromion. The shock was certainly not so great, but I feel that Berger's operation would have been the better procedure.

The man received seven exposures to $X$ rays in as many months, the limited number being due to the distance at wnich he lived. He reports that be is in perfeet health at present. 